\title{
EFECTO HEPATOPROTECTOR DEL GEL DE Aloe vera L. MODIFICADO FRENTE A LA INJURIA INDUCIDA EN RATONES CON ACEITE COMESTIBLE RECALENTADO MEZCLADO CON DMSO
}

\author{
Hepatoprotective effect of modified Aloe vera gel to lesions induced in mice with mixed \\ overheated cooking oil with DMSO
}

Félix Saavedra ${ }^{1}$, Gloria Gordillo² ${ }^{2}$ Haydée Zúñiga², José Apesteguía ${ }^{3}$, Henry Ostos ${ }^{4}$

${ }^{1}$ Instituto de Investigación en Ciencias Farmacéuticas y Recursos Naturales "Juan de Dios Guevara”; ${ }^{2}$ Instituto de Química Biológica, Microbiología y Biotecnología "Marco Antonio Garrido Malo”; ${ }^{3}$ Laboratorio de Toxicología y Química Legal; ${ }^{4}$ Bioterio Facultad de Farmacia y Bioquímica. Universidad Nacional Mayor de San Marcos.

\section{RESUMEN}

El objetivo del presente estudio fue evaluar el efecto hepatoprotector del gel de Aloe vera modificado (GAVM) frente a la injuria inducida en ratones albinos por la administración, vía oral, de aceite comestible recalentado (ACR) mezclado con dimetilsulfóxido (DMSO) (1:1). Las dosis de ACR usadas fueron 2,3y3,1 mL/kg, administradas por 14 días, locual produjo incrementode las transaminasas (ALT, AST) y triglicéridos. A los ejemplares que recibieron la menor dosis de ACR $(2,3 \mathrm{~mL} / \mathrm{kg}$ de peso), a partir del día 14 y por 18 días, se les suministró, adicionalmente, cuatro gotas diarias de GAVM equivalente a $6,1 \mathrm{~mL} / \mathrm{kg}$ de peso. Al analizar los resultados obtenidos a los 14, 19, 28 y 33 días de iniciado el tratamiento, se observó disminución progresiva significativa de ALT, AST y triglicéridos. Sin embargo, los ejemplares que recibieron mayor dosis de ACR (3,1 mL/kg de peso) más GAVM, presentaron incremento en los niveles de ALT, AST y triglicéridos conforme transcurrió el tiempo, sugiriendo que la peroxidación lipídica y los radicales libres formados rebasan el efecto hepatoprotector del GAVM. Los niveles de proteínas no experimentaron variaciones significativas. Los ratones de tres grupos control no presentaron variaciones significativas en los indicadores bioquímicos estudiados, sin embargo, en el grupo control que recibió GAVM se observó disminución de triglicéridos. En conclusión, el GAVM mostró efecto hepatoprotector ante una dosis 2,3 mL/ $\mathrm{kg}$ de peso de ACR, no sucediendo lo mismo al aumentar el ACR (3,1 mL/kg de peso), ante lo cual la dosis de GAVM fue insuficiente.

Palabras clave: Hepatoprotector, Aloe vera, aceite comestible recalentado, dimetilsulfóxido.

\section{SUMMARY}

The aim of this study was to evaluate the hepatoprotective effect of Aloe vera modified gel (MAVG) against the injury induced in albino mice by the oral administration of mixed overheated cooking oil (OCO) with dimethyl sulfoxide (DMSO) (1:1). OCO doses used were 2,3 and $3,1 \mathrm{~mL} / \mathrm{kg}$ administered for 14 days, which produced increases of transaminases ALT, AST and triglycerides. For the specimens that received the lowest dose of OCO $(2,3 \mathrm{~mL} / \mathrm{kg}$ body weight), since day 14 and continuing for 18 days, were administered additionally four drops daily of MAVG equivalent to $6,1 \mathrm{ml} / \mathrm{kg}$. When analyzing the results obtained at $14,19,28$ and 33 days of starting treatment were observed significant progressive decrease in ALT, AST and triglycerides. However, the specimens that received the highest dose of OCO $(3,1 \mathrm{ml} / \mathrm{kg})$ plus MAVG, showed increase in the levels of ALT, AST and triglycerides over time, suggesting that the lipid peroxidation and free radicals formed exceed the hepatoprotective effect of MAVG. The protein levels did not change significantly. The mice of three control groups did not show significant variation in the biochemical indicators studied, however in control group that received MAVG, was observed a decrease of triglycerides. In conclusion, the MAVG presented hepatoprotective effect with a dose of OCO to 2,3 mL/Kg of weight, but it does not happen the same when was increased OCO (3,1 ml / kg of weight ), where the dose of MAVG was insufficient beacause MAVG the dose became insufficient.

Keywords: Hepatoprotective, Aloe vera, overheated cooking oil .

\section{INTRODUCCIÓN}

$\mathrm{s}$ conocido que los aceites vegetales son necesarios para la salud por contener ácidos grasos no saturados. Su insuficiencia en la dieta altera el metabolismo del colesterol, lo que es uno de los principales factores en el desarrollo de la aterosclerosis. Estos trastornos se inician en la juventud y después de algunas décadas pueden ocasionar ataques al corazón o accidentes cardiovasculares.

Los aceites comestibles recalentados originan aldehídos oxigenados $\alpha, \beta$ insaturados-como por ejemplo 4-hidroxi-trans-2-nonenal-, los cuales pueden ser ingeridos a través de la dieta. Además, han sido descritos por primera vez en alimentos otros dos aldehídos de este tipo (4-oxo-trans-2-decenal y 4-oxo-trans-2-undecenal), 
que solamente habían sido objeto de investigaciones biomédicas, demostrándose que su presencia en el organismo está relacionada con diferentes tipos de tumores y el desarrollo de enfermedades neurodegenerativas como el síndrome del Alzheimer $y$ la enfermedad de Parkinson. Todos estos aldehídos son

Tabla 1. Valores de las concentraciones de indicadores bioquímicos en ratones por efecto de GAVM frente a la injuria con ACR.

\begin{tabular}{|c|c|c|c|c|c|c|c|c|}
\hline Grupos & $\begin{array}{l}\text { Sustancia } \\
\text { administrada } \\
\text { por vía oral }\end{array}$ & $\begin{array}{l}\text { Tiempo de } \\
\text { administración } \\
\text { (Días) }\end{array}$ & $\begin{array}{l}\text { Peso } \\
(\mathrm{g})\end{array}$ & $\begin{array}{l}\text { AST } \\
(\mathrm{U} / L)\end{array}$ & $\underset{(\mathbf{U} / L)}{A L T}$ & $\begin{array}{l}\text { Proteínas } \\
\text { (g/dL) }\end{array}$ & $\begin{array}{l}\text { Fosfatasa } \\
\text { Alcalina } \\
\text { (U/L) }\end{array}$ & $\begin{array}{l}\text { Triglicéridos } \\
\text { (mg/dL) }\end{array}$ \\
\hline \multirow{3}{*}{ A } & \multirow{3}{*}{$\begin{array}{l}\text { ACS }(2,3 \mathrm{~mL} / \mathrm{Kg}) \\
+ \text { DMSO }(1: 1)+ \\
\operatorname{GAVM}(6,1 \mathrm{~mL} / \mathrm{Kg})\end{array}$} & 19 & 31 & 232 & 245 & 8,5 & 278 & 278 \\
\hline & & 28 & 33 & 198 & 205 & 8,7 & 259 & 261 \\
\hline & & 33 & 34 & 129 & 131 & 7,8 & 191 & 184 \\
\hline \multirow{3}{*}{ B } & \multirow{3}{*}{$\begin{array}{c}\operatorname{ACS}(3,1 \mathrm{~mL} / \mathrm{Kg}) \\
+ \text { DMSO }(1: 1)+ \\
\operatorname{GAVM}(6,1 \mathrm{~mL} / \mathrm{Kg})\end{array}$} & 19 & 31 & 168 & 167 & 7,9 & 199 & 168 \\
\hline & & 28 & 29 & 169 & 155 & 8,3 & 214 & 301 \\
\hline & & 33 & 34 & 206 & 201 & 8,5 & 237 & 233 \\
\hline \multirow{3}{*}{$\mathrm{C}$} & \multirow{3}{*}{$\begin{array}{c}\text { GAVM } \\
(6,1 \mathrm{~mL} / \mathrm{Kg})\end{array}$} & 14 & 31 & 55 & 73 & 7,6 & 225 & 67 \\
\hline & & 19 & 33 & 61 & 54 & 7,3 & 166 & 45 \\
\hline & & 33 & 34 & 45 & 42 & 7,6 & 148 & 87 \\
\hline \multirow{3}{*}{$\mathrm{D}$} & \multirow{3}{*}{$\begin{array}{c}\text { Control + DMSO } \\
(3 \mathrm{~mL} / \mathrm{Kg})\end{array}$} & 6 & 27 & 67 & 78 & 6,5 & 216 & 198 \\
\hline & & 28 & 35 & 48 & 53 & 7,3 & 155 & 79 \\
\hline & & 33 & 33 & 45 & 49 & 7 & 155 & 104 \\
\hline \multirow{3}{*}{$\mathrm{E}$} & Control: & 6 & 35 & 50 & 69 & 6,9 & 235 & 85 \\
\hline & alimentos $+\mathrm{H}_{2} \mathrm{O}$ & 19 & 36 & 48 & 52 & 7,3 & 155 & 142 \\
\hline & ad libitum & 33 & 39 & 67 & 98 & 7,8 & 186 & 55 \\
\hline
\end{tabular}

muy reactivos y pueden interaccionar con proteínas, hormonas, enzimas y otros componentes del organismo, modificando su estructura y en ocasiones su función ${ }^{(1)}$.

Otros investigadores administraron oralmente Aceites dietéticos oxidados (manteca de cerdo, aceite de soja y aceite de sardina) a ratones machos línea $\mathrm{C}_{3} \mathrm{H}$ HeN, llegando a demostrar después de doce meses carcinoma maligno hepatocelular y hepatoblastoma, además de tumor benigno desarrollado a los seis meses. El aceite de sardina oxidado presentó los niveles mas altos de 8-hidroxi-deoxiguanosina (8-OH-dG) hepática, malonaldehído (MA) y glioxal, a los cuales se atribuye la incidencia del tumor maligno ${ }^{(2)}$.

Tambiénasidoestudiadalaformacióndecompuestos reactivos carbonilo (CRC) en aceites dietéticos oxidados calentados a $60^{\circ} \mathrm{C}$ y $200^{\circ} \mathrm{C}$ por períodos variables- hasta por doce meses y además la toxicidad de estos aceites. Los aceites de salmón y sardina produjeron cantidades mas altas de los aldehídos malonaldehido (MA), glioxal y metilglioxal que los vegetales, sugiriendo que estos compuestos poseen efecto carcinogénico en el hígado de animales de experimentación. También se investigó algunos antioxidantes fitoquímicos como sesamol, eugenol y vinilguayacol, los cuales mostraron actividad antioxidante comparable a BHT y $\alpha$-tocoferol en una concentración $10 \mu \mathrm{g} / \mathrm{mL}$, pudiendo además impedir la formación de CRC; sin embargo, los antioxidantes mencionados pueden tener propiedades oxidativas en la peroxidación lipídica ${ }^{(3)}$.

En un estudio, realizado por Abilés y col., aceites de maíz, oliva virgen y grasa de vaca calentados a $18 \mathrm{o} \pm 2^{\circ} \mathrm{C}$ durante siete minutos fueron administrados a ratas Winstar durante treinta días. Se cuantificó en suero la concentración de malonilaldehído (MA) por el método del ácido tiobarbitúrico (ATB), el cual al reaccionar con una molécula de MA y muchos aldehídos $\alpha$ y $\beta$ insaturados biológicamente activos y procedentes de la peroxidación lipídica (cuyos productos finales pueden ser mutagénicos y carcinogénicos), forma un cromógeno rosa, medible por espectrofotometría. El experimento demostró que la peroxidación lipídica plasmática es mayor cuanto mayor sea el número de calentamientos aplicados. También se afectaron los niveles de colesterol, incrementándose la colesterolemia, sobretodo en los roedores que consumieron grasa de vaca ${ }^{(4)}$.

Koch et al., observaron que El aceite de girasol calentado a $60^{\circ} \mathrm{C}$ durante 25 días y administrado a ratas durante seis días causa un incremento significativo de productos de peroxidación, dienos conjugados, carbonilos totales y TBARS. Asimismo, se incrementaron las concentraciones relativas de mRNA de los genes acyl-CoA oxidasa (ACO) y citocromo $\mathrm{P} 450$ 4A1 (Cyp4A1) respecto a los roedores que consumieron aceite fresco ${ }^{(5)}$.

Otro estudio reveló que Los ratones que recibieron en su dieta aceite de girasol termo oxidado -después de calentar papas quince veces por veinte minutos--, durante un mes, presentaron aberraciones cromosómicas y fragmentación de ADN en su esperma, estas alteraciones disminuyen cuandoal aceite oxidado se añadieron rodajas de tomate y zanahorias -previamente deshidratadas- en proporción 5 y $10 \%$ respecto a su dieta total ${ }^{(6)}$.

Losaceitesfrescosdesojaypalmanosonperjudiciales para la presión sanguínea, significativamente elevan 
los niveles de óxido nítrico y reducen las respuestas de contracción. Sin embargo, cuando son calentados de manera repetida -durante el proceso de cocina-, generan radicales libres y reducen los niveles de antioxidantes y vitaminas, lo cual conduce al estrés oxidativo. El incremento de especies reactivas del oxigeno (ROS, por sus siglas en inglés) y el balance alterado entre óxido nítrico $(\mathrm{NO})$ y compuestos oxigenados reactivos, conduce a una deteriorada biodisponibilidad de NO, que resulta en una disminución vasorelajante endotelio-dependiente que conduce a hipertensión ${ }^{(7)}$.

En nuestro medio es común el consumo de frituras en locales públicos donde es visible el uso de aceite comestible recalentado (ACR), por este motivo, se diseño un modelo sencillo para evaluar el efecto hepatoprotector del gel de Aloe vera modificado (GAVM), en ratones albinos intoxicados con ACR.

\section{MATERIALES Y MÉTODO}

\section{Preparación de las mezclas oleosas}

La muestra de aceite comestible recalentado (ACR) se obtuvo de un establecimiento ambulatorio de venta de pollo fritoal paso. Esteaceiteporsu reiteradocalentamiento tenía aspecto oscuro y finas partículas en suspensión.

Con la finalidad de favorecer la dilución del aceite y suadministración per os a los ratones deexperimentación, se utilizó dimetilsulfoxido (DMSO) en la proporción 1:1. El DMSO es un disolvente de aspecto oleoso.

\section{Reactivos químicos}

Todos los reactivos utilizados fueron grado analítico.

\section{Animales de experimentación}

Se utilizaron ratones albinos hembras, cepa $\mathrm{N}_{1} \mathrm{H}$, del Instituto Nacional de Salud con peso inicial de 20-25 g, aclimatados por siete días, con acceso libre a su alimento y agua, bajo condiciones estables de temperatura $\left(23^{\circ} \mathrm{C}\right)$ y humedad $(60-70 \%)$. Los roedores se dividieron en cinco grupos de cinco cada uno y el tratamiento oral fue por 32 días. Peso promedio a partir de los primeros sacrificios $33 \mathrm{~g}$.

Grupo A: recibieron por vía oral 3 gotas de la mezcla ACR+DMSO (1:1) equivalente a ACR 2,3 mL/Kg de peso durante 14 días. Luego por 18 días más, sin suspender la ingesta de ACR, se les administró IV gotas de GAVM (6,1 mL/Kg de peso).

Grupo B: recibieron por vía oral 4 gotas de la mezcla ACR+DMSO (1:1) equivalente a ACR 3,1 mL/Kg de peso durante 14 días. Luego por 18 días, sin suspender la ingesta de ACR, se les administró IV gotas de GAVM $(6,1 \mathrm{~mL} / \mathrm{Kg}$ de peso).

Grupo C: control, recibieron GAVM $(6,1 \mathrm{~mL} / \mathrm{Kg})$ durante 32 días.

Grupo D: control, recibieron 2 gotas de DMSO equivalente a $3 \mathrm{~mL} / \mathrm{Kg}$ de peso por 32 días.

Grupo E: control con acceso libre a alimento y agua por 32 días.

\section{Gel de aloe vera modificado}

Para prepararlo, los pedazos de las hojas de Aloe vera se remojaron en agua destilada por cuatro horas, luego se cambió el agua y nuevamente se dejaron remojar por dos horas, para finalmente separar el gel con espátula para licuarlo y luego guardarlo bajo refrigeración por dos semanas, según procedimiento descrito por Saavedra y col ${ }^{(8)}$.

\section{Preparación de las muestras biológicas}

A los ratones previamente anestesiados, se les extrajeron muestras de sangre mediante punción cardiaca y se separó el suero para las determinaciones de: ALT, AST, triglicéridos, fosfatasa alcalina y proteínas totales por métodos convencionales.

\section{RESULTADOS}

En la tabla 1 se presentan las dosis de ACR y GAVM que se administraron a los grupos investigados A y B durante diferentes periodos; destaca el que presentaran un peso promedio semejante al de los tres grupos control.

Se determinaron los niveles de ALT, AST, fosfatasa alcalina, triglicéridos y proteínas totales en los grupos A y $\mathrm{B}$ a los 19, 28 y 33 días después de iniciado el tratamiento con GAVM con la finalidad evaluar su rol hepatoprotector en animales a los que al mismo recibieron dosis variable de ACR. En las figuras 1 y 2 se observan las variaciones de dichos indicadores en los grupos A y B; además, se observa la variación de los mismos para los tres grupos control C, D y E, medidos en diferentes momentos luego de iniciado el experimento.

En la figura 1 puede observarse que los grupos $\mathrm{A}$ y B que recibieron la mezcla ACR + DMSO durante 14 días, luego de cinco días de tratamiento con GAVM presentan niveles de transaminasas (AST y ALT) bastante altos respecto a los ejemplares de los grupos control C, D y E. La fosfatasa no se presenta igualmente alta; sugiero agregar: La fosfatasa alcalina también estuvo elevada en el grupo A, pero no tuvo igual comportamiento en el grupo $\mathrm{B}$. 
El grupo A, que recibió menor cantidad de ACR, presentó disminución de los indicadores bioquímicos mencionados conforme transcurrieron los días de tratamiento con GAVM; sin embargo, el grupo $B$ que recibió mayor cantidad de ACR y la misma

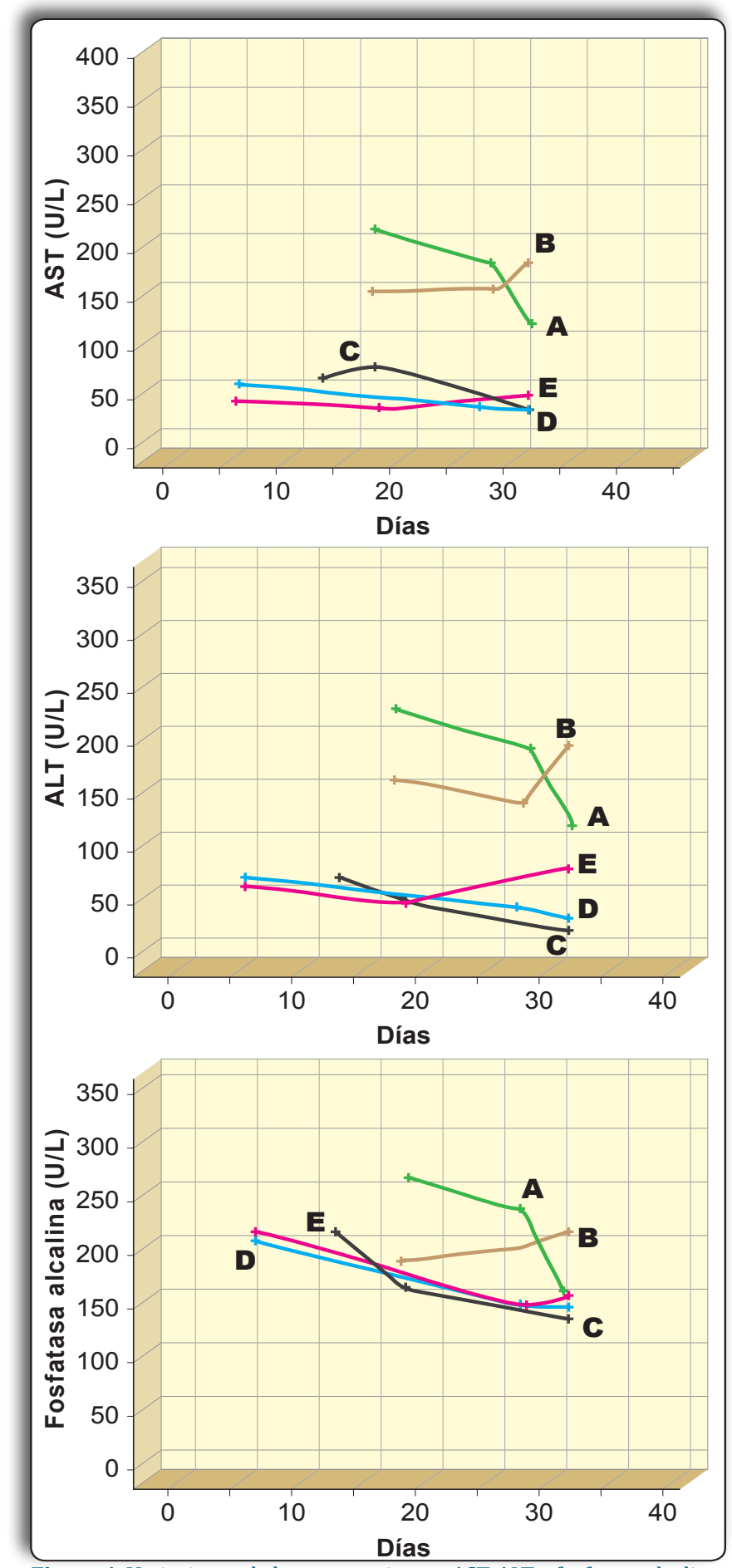

Figura 1. Variaciones de las transaminasas AST, ALT y fosfatasa alcalina. dosis de GAVM mostró incremento de los niveles de transaminasas y fosfatasa alcalina (figura 1).

Con relación a los triglicéridos y proteínas plasmáticas (figura 2), se observó un comportamiento similar; conforme transcurre el tiempo, tienden a disminuir en el grupo A y se incrementan en el grupo B.

Por otro lado, los ejemplares de los grupos A y B sacrificados a los 19 días (05 días de tratamiento con GAVM), tuvieron vísceras de color rojo oscuro, especialmente los intestinos; a nivel hepático se observó la formación de vesículas oleosas amarillentas (figura 3), las cuales disminuyeron de tamaño y en algunos casos desaparecieron conformeavanzó el tiempo de tratamiento. Los roedores sacrificados a los 28 y 33 días lucían órganos internos (incluyendo el hígado) sanos y bien irrigados.

Los roedores del grupo control C, al cual se administró GAVM $(6,1 \mathrm{ml} / \mathrm{Kg})$ durante todo el tratamiento, mostraron disminución de los triglicéridos respecto a los otros dos grupos control D y E, también presentaron enrojecimiento en el orificio anal y frecuente evacuación de heces y orina; sin embargo, no experimentaron disminución de peso en comparación con los demás grupos. Cuando los ratones de los tres grupos control fueron sacrificados en ayunas, sus órganos internos presentaron buen color e irrigación sanguínea normales.

\section{DISCUSIÓN}

El efecto hepatoprotector del gel de Aloe vera se ha demostrado con diversos estudios, por ejemplo: las dosis $250 \mathrm{mg} / \mathrm{Kg}$ o $500 \mathrm{mg} / \mathrm{Kg}$ disminuyeron el incremento de ALT, AST y fosfatasa alcalina producidos por la hepatotoxicidad inducida por paracetamol dosis $3 \mathrm{~g} / \mathrm{Kg}$ de peso en ratas albinas ${ }^{(9)}$.

También se ha sugerido que el extracto acuoso de las hojas de Aloe vera tiene efecto hepatoprotector en ratones con diabetes inducida por aloxano, puesto que ocasionan la disminución de los niveles de ALT, AST, bilirrubina y glucosa, incrementados por la administración del compuesto injuriante ${ }^{(10)}$.

Asimismo, otro estudio reportó que el extracto acuoso presenta efecto hepatoterapéutico en ratas intoxicadas con etanol, lo que se evidencia al correlacionar la mejoría en la función hepática con el estudio histopatológico después de la administración de Aloe vera. Los indicadores bioquímicos evaluados fueron bilirrubina sérica total, fosfatasa alcalina, ALT y AST ${ }^{(11)}$.

Con la finalidad de disminuir los efectos dañinos de los productos generados por el calentamiento repetido de 


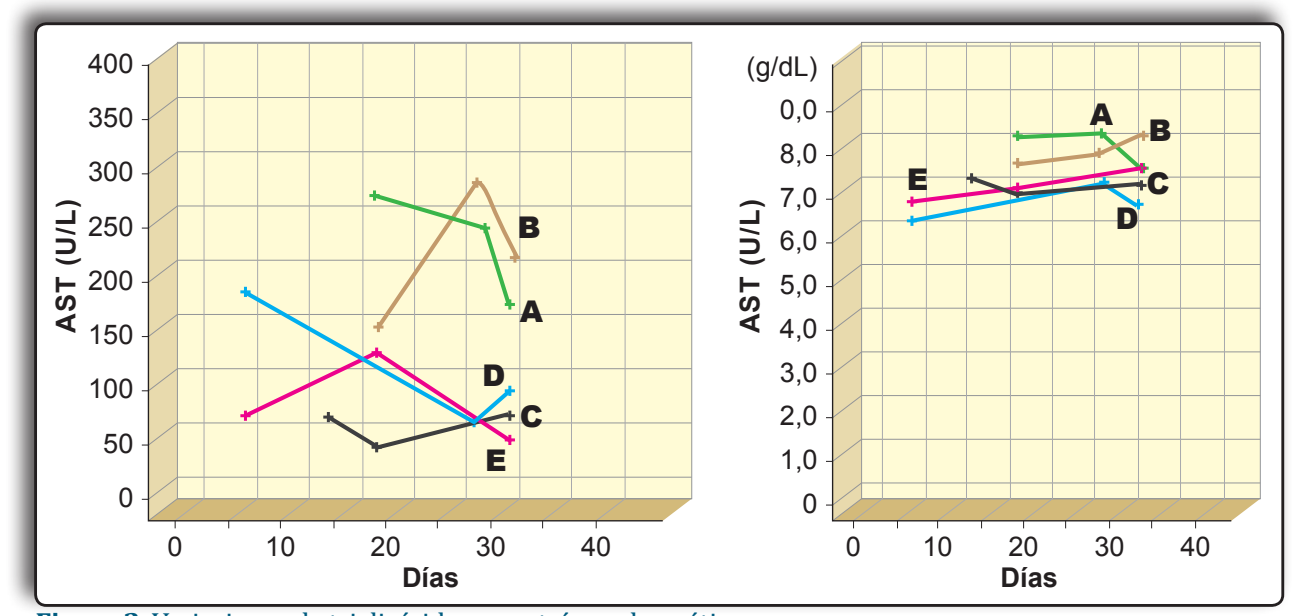

Figura 2. Variaciones de triglicéridos y proteínas plasmáticas.

aceites comestibles, a veces a temperaturas cercanas a los $175-185^{\circ} \mathrm{C}^{(12)}$, se han realizado estudios para demostrar las bondades del arte culinario, así el aceite de soja calentado a $200^{\circ} \mathrm{C}$ por cinco días, durante dos horas y añadido a la dieta de ratas durante cuatro semanas, produjo un aumento significativo del valor de peróxido y malondialdehido respecto a los roedores que consumieron aceite fresco sin calentar-, sin embargo, cuando al aceite calentado se añadió cebolla y ajos en trozos ( o,1 y o,2 g/mL de aceite), los indicadores mencionados fueron significativamente menores respecto a los animales que consumieron el aceite calentado sin aditivos. Similar resultado se obtuvo con las enzimas ALT y AST, siendo mayores las concentraciones cuando se consumió aceite calentado que con la ingesta de aceite calentado con dichos aditivos ${ }^{(13)}$.

El GAVM ha demostrado efecto hepatoprotector en ratas intoxicadas con tetracloruro de carbono al presentarse disminución de ALT, AST, fosfatasa alcalina y triglicéridos ${ }^{(8)}$. En el presente estudio en ratones intoxicados con aceite comestible recalentado (ACR), se evaluó el rol hepatoprotector del GAVM, comprobando la variación de los indicadores mencionados.

De acuerdo a la figura 1 se observa que el aceite comestible recalentado eleva de manera muy significativa el contenido de las enzimas ALT y AST con relación a los grupos control (ver tabla 1); mientras que con la administración de GAVM durante 18 días, dosis $6,1 \mathrm{~mL} / \mathrm{Kg}$ de peso, se logra disminuir de manera notable los niveles de dichas enzimas, sugiriendo su efecto hepatoprotector.

Cuando los aceites comestibles y dietéticos son oxidados por calentamiento repetido y se administran a animales de experimentación, se presentan efectos perjudiciales, así tenemos: el consumo prolongado de aceite de soja termo oxidado -calentado diez días, por dos horas-, administrado durante seis semanas a ratas, produjo una visible disminución de peso corporal y fosfatasa alcalina, respecto a los roedores que consumieron la misma cantidad de aceite fresco en su dieta; sin embargo, el contenido tisular de proteínas no es significativo para ambos grupos ${ }^{(14)}$. La ingesta de GAVM a los ratones logra disminuir la fosfatasa alcalina y no es significativo el contenido tisular de proteínas en los grupos que recibieron ACR y los tres grupos control.

Por efecto del GAVM disminuyeron los niveles de los cuatro primeros indicadores mencionados (figuras 1 y 2) y las vesículas oleosas disminuyeron progresivamente de tamaño desapareciendo en los últimos ejemplares sacrificados.

En el grupo A, que recibió menor cantidad de $\operatorname{ACR}(2,3 \mathrm{~mL} / \mathrm{Kg})$, fue evidente la disminución de transaminasas AST, ALT, fosfatasa alcalina, triglicéridos y proteínas totales por efecto del GAVM $(6,1 \mathrm{~mL} / \mathrm{Kg})$; pero, en el grupo $B$, que recibió mayor cantidad de ACR $(3,1 \mathrm{~mL} / \mathrm{Kg})$, los niveles de estos metabolitos continuaron incrementándose, haciéndose evidente

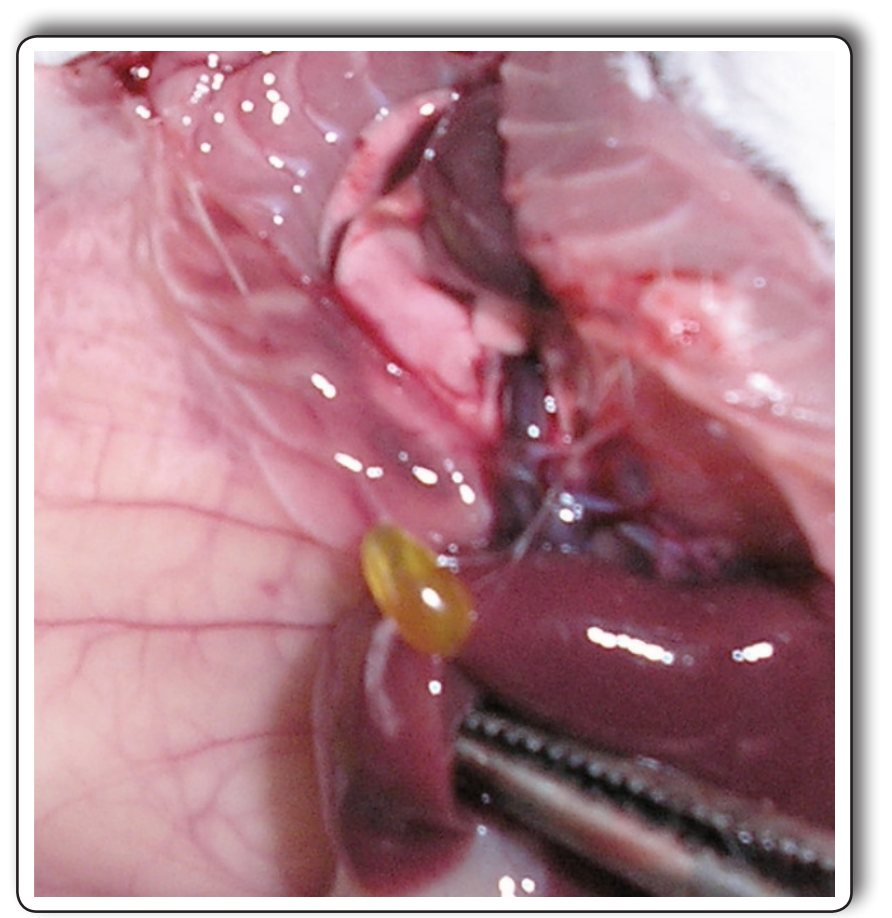

Figura 3. Vesícula oleosa sobre el hígado 
que la cantidad de GAVM $(6,1 \mathrm{~mL} / \mathrm{Kg})$ fue insuficiente para ejercer su efecto hepatoprotector.

\section{CONCLUSIONES}

1. El aceite comestible recalentado (ACR), administrado a ratones por vía oral, aumenta los niveles de ALT, AST y triglicéridos.

2. El gel de Aloe vera modificado $6,1 \mathrm{~mL} / \mathrm{Kg}$, administrado al grupo que recibió menor cantidad de ACR $(2,3 \mathrm{~mL} / \mathrm{Kg})$ disminuyó los niveles de los indicadores ALT, AST, triglicéridos y proteínas plasmáticas, demostrando efecto hepatoprotector.

3. El grupo que recibió mayor cantidad de ACR (3,1 $\mathrm{mL} / \mathrm{Kg})$ y la misma cantidad de GAVM $(6,1 \mathrm{~mL} /$ $\mathrm{Kg}$ ) no mostró efecto hepatoprotector, a juzgar por los mayores niveles de ALT, AST, fosfatasa alcalina, triglicéridos y proteínas encontrados.

\section{REFERENCIAS BIBLIOGRÁFICAS}

1. Guillén MD, Uriarte PS. Aldehydes contained in edible oils of a very different nature after prolonged heating at frying temperature: Presence of toxic oxygenated $\alpha, \beta$ unsaturated aldehydes. Food Chemistry. 2012; 131(3): 91526.

2. Ichinose T, Nobuyuki S, Takano H, Abe M, Sadakane K, Yanagisawa R, et al. Liver carcinogenesis and formation of 8-hydoxy-deoxyguanosine in $\mathrm{C}_{3} \mathrm{H} / \mathrm{HeN}$ mice by oxidized dietary oils containing carcinogenic dicarbonil compounds. Food Chem Toxicol. 2004; 42(11): 1795-803.

3. Fujiota K. Formation/inhibition and toxicity of reactive carbonyl compounds from oxidized dietary oils: Antioxidant properties of phytochemicals measured by headspace solid-phase microextraction/ gas chromatography. Agricultural and Environmetal Chemistry. University of California. Davis; 2005.

4. Abilés J, Ramón AN, Moratalla G, Pérez-Abud R, Morón Jiménez J, Ayala A. Efectos del consumo de aceites termooxidados sobre la peroxidación lipídica en animales de laboratorio. Nutrición Hospitalaria. 2009; 24(4): 1-9.

5. Koch A, König B, Spielmann J, Leitner A, Stangl GI, Eder $\mathrm{K}$. Thermally oxidized oil increases the expression of insulin-induced genes and activation of sterol regulatory element-binding protein-2 in rat liver. Journal of Nutrition. 2007; 137(9): 2018-23.

6. Eshak MG, Ghaly IS, Khalil WK, Farag IM, Ghanem KZ. Genetic alterations induced by toxic effect of thermally oxidized oil and protective role of tomatoes and carrots in mice. Journal of American Science [Internet]. 2010 [Citado 10 nov 2014]; 6(4): 175-86. Disponible en: http://
www.researchgate.net/publication/228347022_Genetic _ alterations_induced_by_toxic_effect_of_thermally_ oxidized_oil_and_protective_role_of_tomatoes_and_ carrots_in_mice

7. Jaarin K, Mustafa MR, Leong XF. The effects of heated vegetable oils on blood pressure in rats. Clinics (Sao Paulo). 2011; 66(12): 2125-32.

8. Saavedra F, Gordillo G, Zúñiga H, Apesteguia J, Ostos H. Efecto del gel de Aloe vera $\mathrm{L}$. modificado en el tratamiento de hepatotoxicidad inducida con $\mathrm{CCl}_{4}$ y $\mathrm{DMSO}$ en ratones. Ciencia e Investigación. 2013; 16(1): 41-7.

9. Nayak V, Gincy TB, Prakash M, Joshi Ch, Soumya S, et al. Hepatoprotective activity of Aloe vera gel against paracetamol induced hepatotoxicity in albino rats. Asian J Phar Biol Res. 2011; 1(2): 94-8.

10. Sharma B, Siddiqui S, Ram G, Chaudhary M, Sharma G. Hypoglycemic and hepatoprotective effects of processed Aloe vera gel in a mice model of alloxan induced diabetes mellitus. J Diabetes Metab [Internet]. 2013 [Citado 21 abril 2015]; 4(9). Disponible en: http://omicsonline. org/hypoglycemic-and-hepatoprotective-effects-ofprocessed-aloe-vera-gel-2155-6156.1000303.pdf

11. Saka WA, Akhigbe RE, Ishola OS, Ashamu EA, Olayemi OT, Adeleke GE. Hepatotherapeutic effect of Aloe vera in alcohol-induced hepatic damage. Pakistan Journal of Biological Sciences. 2011; 14(14): 742-6.

12. Yagüe MA. Estudio de utilización de aceites para frituras en establecimientos alimentarios de comidas preparadas. [Internet]. Bellaterra: UAB; 2003. Disponible en: https:// avdiaz.files.wordpress.com/2008/o8/mangeles-aylonblog.pdf

13. Oyewole OI, Olayinka ET. Protective role of onion and garlic on physicochemical alterations and toxicity of heated soybean oil. African Journal of Biotechnology. 2007; 6(18): 2158-61.

14. Odotuga AA, Oyewole OI.. Effect of thermo-oxidized soybean oil on growth rate and skeletal muscle enzyme activities in rats. Biokemistri 2006; 18(1): 9-13. [En línea]. Acceso 21 abril 2015. Disponible en www.bioline.org.br/ request?bko6oo2

Manuscrito recibido el: $26 / 11 / 14$

Aceptado para su publicación el: 20/05/2015

\section{Correspondencia}

Nombre: $\quad$ Félix E. Saavedra Nizama

Dirección: Jr. Puno 1002. Lab. Química Inorgánica

E-mail: fsaavedran@unmsm.edu.pe

fesn2709@gmail.com 